\title{
SARCOID CARDIOMYOPATHY
}

\author{
Sivakumar Srinivasan, MD and Emanuel Chryssos, MD
}

The patient is a 40 year old African-American female with no significant past medical history who presented to the ED with a 2 week history of lower extremity edema and shortness of breath. She also noticed intermittent palpitations, dry cough, easy fatigue and a 30 pound weight loss. On examination, the patient's temperature was $98.9 \mathrm{~F}$ orally, pulse was $155-170$ beats per minute, and blood pressure was $133 / 93 \mathrm{mmHg}$. EKG revealed long P-R tachycardia with heart rate of 170 . Chest X-ray was done and showed large right pleural effusion with compressive atelectasis and small left pleural effusion. Transthoracic echocardiogram at the time of admission was consistent with severe global LV dysfunction with EF-20\% and moderate pulmonary hypertension. CT Chest, which showed bilateral hilar adenopathy and subsequent biopsy was consistent with non-caseating granulomatous lesion. Her initial basic laboratory values, including TSH level, were normal except for BNP which was 569. She underwent left and right heart catheterization, which revealed normal coronary arteries and decreased cardiac index. During the hospital stay, telemetry monitors revealed rhythms consistent with various degrees of AV conduction abnormalities. She was started on steroids and intravenous milrinone for possible sarcoid cardiomyopathy and listed for heart transplant. Her symptoms were gradually improved and she was discharged home with steroids, beta blockers, diuretics, ace inhibitors and an implantable defibrillator. She is currently followed as an outpatient and doing extremely well without any cardiac symptoms.

Myocardial sarcoidosis may have restrictive as well as congestive features because cardiac infiltration by sarcoid granulomas results not only in increased stiffness of the ventricular wall but also in diminished systolic contractile function. Occasionally patients with extensive involvement of the left ventricular myocardium develop left ventricular aneurysms; it has been suggested that corticosteroid treatment may convert granulomas to scar tissue and contribute to the development of aneurysmal dilatation. Firstdegree heart block due to disease of AV Node or bundle of His, and various types of intraventricular conduction defects, are common among patients with cardiac sarcoidosis. Complete heart block is the most common finding in patients with clinically evident cardiac sarcoidosis, and occurs at a younger age in patients with sarcoidosis than in individuals with complete heart block due to other etiologies. Corticosteriods can be used to halt progression of disease, but it has little to no benefit once EF is $<30 \%$. Heart transplantation for sarcoidosis with advanced heart failure has been performed less commonly than lung transplantation. Survival has been similar to that of patients undergoing heart transplantion for other indications. Despite the possibility of recurrence of sarcoidosis in the graft or the appearance of progressive extracardiac disease, available evidence supports transplantation for cardiac sarcoidosis in patients with extensive cardiac involvement and advanced heart failure as long as there is minimal extracardiac involvement.

Credits: H\&E stains prepared by Bernadette Wildermore MD

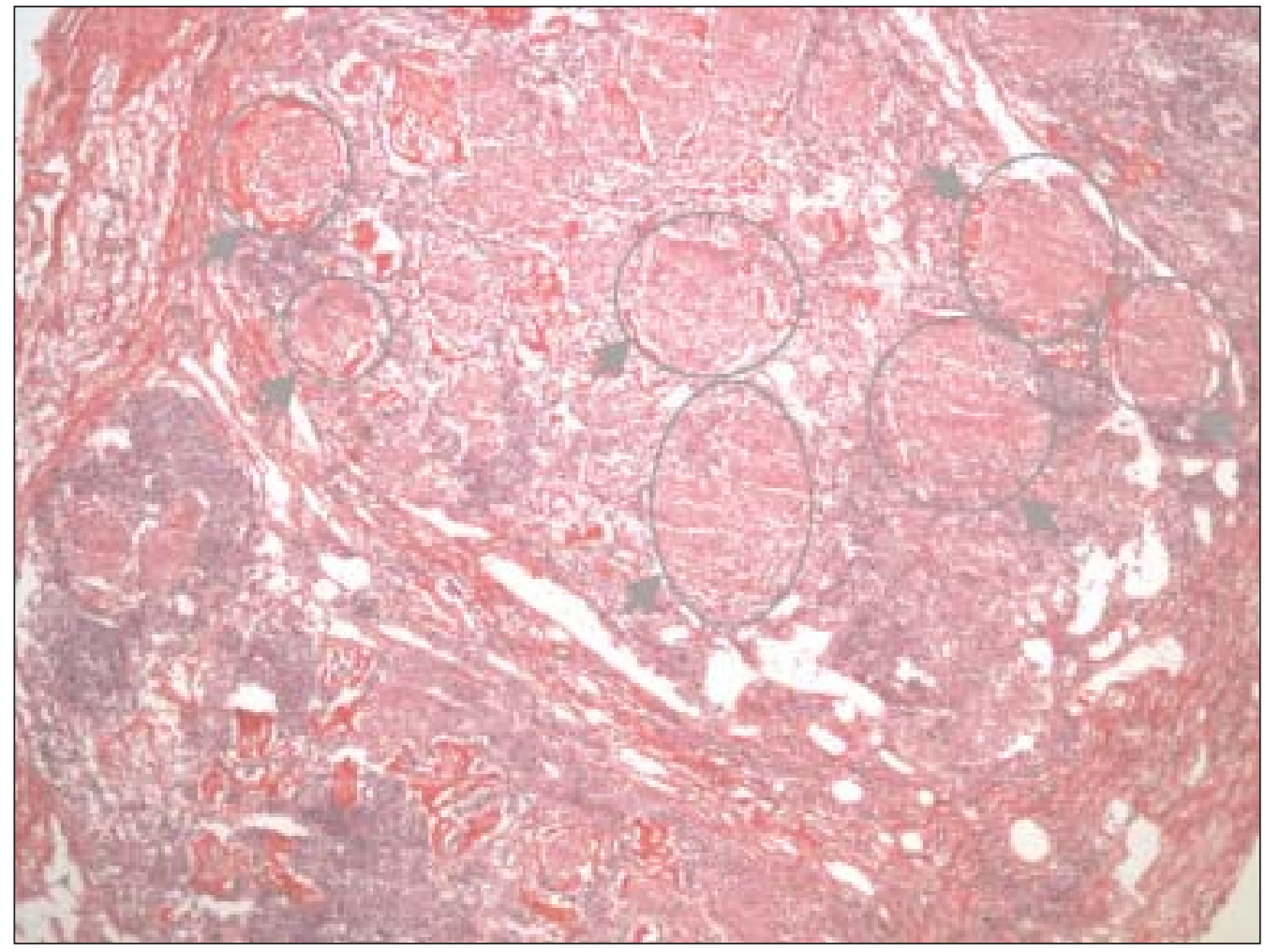

Figure 1. Hematoxylin and Eosin stain of lymph node at low power magnification showing multiple noncaseating granulomas. (arrows) 


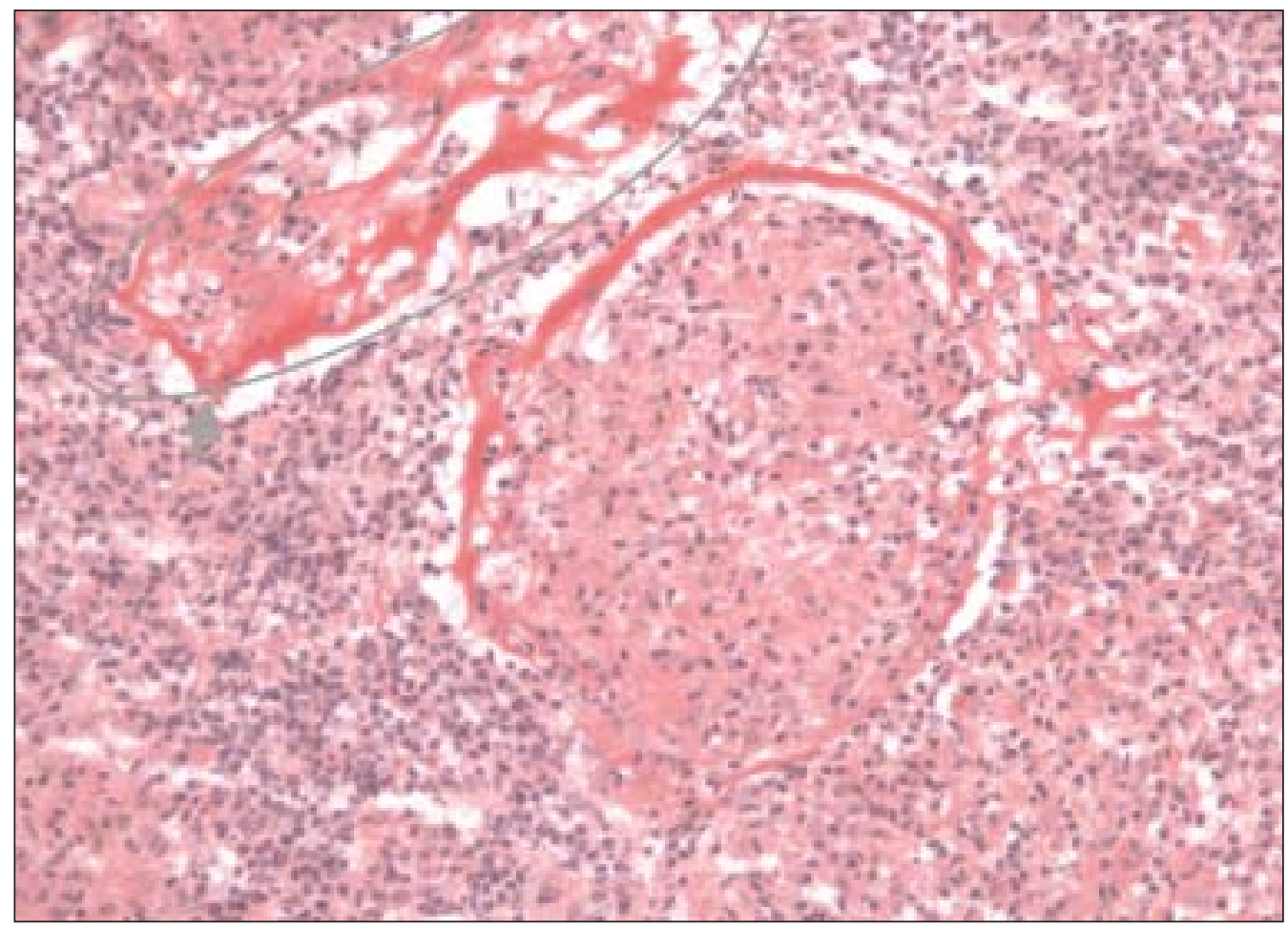

Figure 2. Higher power magnification reveals lack of necrotic material within the granuloma. Also seen is peri granulomatous connective tissue (arrow) consistent with fibrosis which can sometimes be a marker of long-standing sarcoid.

Case Reports

\section{IMAGE IN MEDICINE}

\section{Amy Baranoski, $M D$}

Pictured here is the back of a man with leprosy who had been treated at Acworth Leprosy Hospital in Mumbai, India. Note the diffuse, macular, hypopigmented rash. Leprosy is a chronic disease caused by infection with Mycobacterium leprae. The disease can affect a variety of organs including peripheral nerves, skin, muscle, eye, bone, and testes. Leprosy is diagnosed clinically by the presence of at least one of the following: hypopigmented patches, loss of cutaneous sensation, thickened nerves, and acid-fast bacilli in nasal or skin smears.

The prevalence of leprosy has decreased worldwide due to multidrug therapy and public health programs focused on education and detection of the illness. Multi-drug therapy consists of the bactericidal drugs: rifampin, dapsone, clofazimine, ethionamide and protionamide. Quinolones, minocycline, and clarithromycin are not part of the normal regimen, but can be combined with rifampin, primarily in single lesion leprosy.

In November 2005 Dr. Baranoski spent several weeks observing various clinics and hospitals in Mumbai, India where she captured the following image.

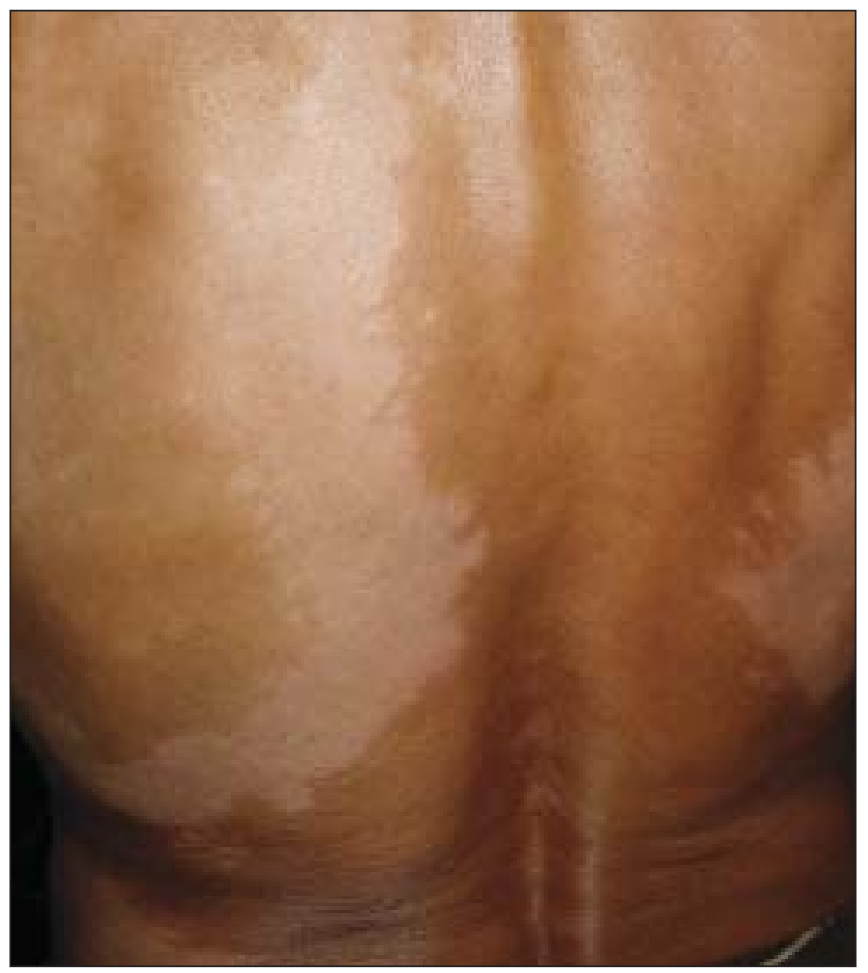

